Proceedings of the 2009 Winter Simulation Conference

M. D. Rossetti, R. R. Hill, B. Johansson, A. Dunkin, and R. G. Ingalls, eds.

\title{
NEWTON-RAPHSON VERSION OF STOCHASTIC APPROXIMATION OVER DISCRETE SETS
}

\author{
Eunji Lim \\ Department of Industrial Engineering \\ University of Miami \\ Coral Gables, FL 33124, U.S.A.
}

\begin{abstract}
This paper considers the problem of optimizing a complex stochastic system over a discrete set of feasible values of a parameter when the objective function can only be estimated through simulation. We propose a new gradient-based method that mimics the Newton-Raphson method and makes use of both the gradient and the Hessian of the objective function. The proposed algorithm is designed to give guidance on how to choose the sequence of gains which plays a critical role in the empirical performance of a gradient-based algorithm. In addition to the desired fast convergence in the first few steps of the procedure, the proposed algorithm converges to a local optimizer with probability one as $n$ goes to infinity with rate $1 / n$ where $n$ is the number of iterations.
\end{abstract}

\section{INTRODUCTION}

When we consider the problem of optimizing a complex system whose performance depends on a parameter, we often find that the system requires simulation as an inevitable means of evaluating its performance. When optimizing such systems over a set of feasible values of a parameter, we need different approaches than the traditional optimization methods in order to account for the errors incurred from measuring the system performance through simulation.

In many practical situations, the set of feasible values of a parameter is finite or countably infinite rather than continuous. For instance, when controlling an inventory of a certain product, the cost function associated with the inventory system is a function of the ordering quantity of the product which can only take on nonnegative integers. When allocating buffers to several stations in a queueing system, the average sojourn time in the system depends on the number of buffers which can only take on nonnegative integers. To optimize such systems over discrete sets, several algorithms such as the simulated annealing method by Gelfand and Mitter (1989), the random search method by Andradottir (1995), the nested partitions method by Shi and Olafsson (2000), the sample average approximation method by Kleywegt et al. (2001), a method balancing the search process and the simulation effort by Lin and Lee (2006), and COMPASS by Hong and Nelson (2006) have been proposed.

Recently, gradient-based methods have been proposed by Lim and Glynn (2006) and Dupăc and Herkenrath (1982) for the case where the parameter space is discrete. The algorithm resembles stochastic approximation which was designed for continuous parameter spaces. Stochastic approximation (Robbins and Monro 1951) is a recursive method which updates $\theta_{n}$, the estimated optimal point at the $n$th iteration, by the following formula:

$$
\theta_{n+1}=\theta_{n}-a_{n} D_{n} .
$$

Algorithm (1) above moves along the direction of negative gradient so that the objective function is reduced in each step. In the discrete counterpart of (1) as proposed in Lim and Glynn (2006), $D_{n}$ mimics the derivative of the objective function by computing the difference of the objective values evaluated at the nearest point of $\theta_{n}$ to the right and the nearest point of $\theta_{n}$ to the left.

When applying a gradient-based algorithm such as (1) in practice, we encounter a problem of choosing the right sequence of gains $\left\{a_{n}\right\}$. To ensure the convergence of (1), $\left\{a_{n}\right\}$ needs to satisfy $\sum a_{n}=\infty$ and $\sum a_{n}^{2}<\infty$. A typical choice of $a_{n}$ is $c / n$ 
for some positive constant $c$. In most practical situations where the computational budget and hence the number of simulation runs is pre-determined, the choice of the sequence of gains $\left\{a_{n}\right\}$ influences the empirical performance of the algorithm. When the gains are too large, the estimates for the optimal solution will bounce around the parameter space whereas when the gains are too small, the estimates for the optimal solution will seem to get stuck at some point. Furthermore, one often has no a priori information on the choice of the sequence of gains.

To illustrate the significance of the choice of $\left\{a_{n}\right\}$, consider the problem of minimizing a cost function $f$ which depends on a parameter $\theta$ in $f(\theta)=\theta^{2}$ for all integers $\theta$. For the purpose of simplicity, assume that the measurement of the objective function $f(\theta)$ is exact, i.e., the problem is deterministic. We assume that the initial point $\theta_{1}$ is 100.10 . Since one has no a priori information on $\left\{a_{n}\right\}$, one needs to guess the values of $a_{1}$ and $a_{n}$. Suppose that one decides to try sequences of gains $a_{n}=5 / n, a_{n}=1 / n$, and $a_{n}=1 / 5 n$, then the trajectories of $\left[\theta_{n}\right]$, the nearest integer to $\theta_{n}$, generated from:

$$
\theta_{n+1}=\theta_{n}-a_{n}\left(f\left(\left\lceil\theta_{n}\right\rceil\right)-f\left(\left\lfloor\theta_{n}\right\rfloor\right)\right)
$$

for each choice of the sequence are as follows:

\begin{tabular}{c|cccccc}
\hline$a_{n}=5 / n$ & 100 & -905 & 3618 & -8441 & 12661 & -12660 \\
\hline$a_{n}=1 / n$ & 100 & -101 & -1 & 0 & 0 & 0 \\
\hline$a_{n}=1 / 5 n$ & 100 & 60 & 48 & 42 & 38 & 35 \\
\hline
\end{tabular}

In (2), $\lceil x\rceil$ is the smallest integer greater than or equal to $x$ and $\lfloor x\rfloor$ is the largest integer less than or equal to $x$. When $a_{n}=5 / n$, the estimated optimal points $\left[\theta_{n}\right]$ tend to bounce around too much whereas when $a_{n}=1 / 5 n$, $\left[\theta_{n}\right]$ tends to converge slowly.

To suggest the choice of $\left\{a_{n}\right\}$, we consider a Newton-Raphson type approach. Recall that the Newton-Raphson method to find the optimal point of $f$ is based on the following recursion:

$$
\theta_{n+1}=\theta_{n}-\frac{f^{\prime}\left(\theta_{n}\right)}{f^{\prime \prime}\left(\theta_{n}\right)}
$$

where $f^{\prime}$ and $f^{\prime \prime}$ are the first and second derivatives of $f$, respectively. Considering that stochastic approximation (1) is asymptotically optimal when $a_{n}=1 /\left(f^{\prime \prime}\left(\theta_{*}\right) n\right)$ where $\theta_{*}$ is the optimal point of $f$ as proven in Chung (1954), we propose the following procedure:

$$
\theta_{n+1}=\theta_{n}-\frac{c}{n} \frac{D_{n}}{H_{n}}
$$

where $D_{n}$ is an estimate of $f^{\prime}(\theta)$ and $H_{n}$ is an estimate of $f^{\prime \prime}\left(\theta_{*}\right)$.

The algorithm (4) resembles the Newton-Raphson method and makes use of both the gradient and the Hessian information of the objective function. It suggests a multiple of the reciprocal of the Hessian as the sequence of gains. In addition to fast convergence in the first few iterations, the proposed algorithm enjoys a nice asymptotic property. It converges to a local optimizer with probability one at rate $1 / n$ where $n$ is the number of iterations. The convergence rate $1 / n$ is induced from the fact that the objective function is defined on a discrete set and hence $E\left(D_{n} / H_{n}\right)$ in (4) stays at a constant value when $\theta_{n}$ gets close to $\theta_{*}$, whereas when a smooth objective function is defined on a continuous set, $E\left(D_{n}\right)$ in (1) converges to zero as $\theta_{n}$ gets close to $\theta_{*}$. Therefore, the convergence rate of (4) differs from the conventional convergence rates that are obtained in continuous cases.

This paper is organized as follows. In Section 2, we present the proposed algorithm and our main results. In Section 3, we provide proofs for the main results. In section 4, we illustrate the empirical behavior of the proposed algorithm.

\section{PROBLEM FORMULATION AND MAIN RESULTS}

Consider the following problem of optimization

$$
\min _{\theta \in Z} f(\theta)=E[X(\theta)],
$$




\section{$\operatorname{Lim}$}

where $Z$ is the set of integers. Suppose that $f(\theta)$ cannot be evaluated exactly, it must be estimated through simulation. Our goal is to generate a sequence of random variables $\left\{\theta_{1}, \theta_{2}, \ldots\right\}$ that converges to the optimal solution $\theta_{*}$ of $f$.

The proposed algorithm proceeds as follows. Choose $\theta_{1}$ randomly from $Z$. Given $\theta_{1}, \ldots, \theta_{n}$, one generates $X_{n}^{+}, X_{n}^{-}, X_{n}^{\prime}$, $X_{n}^{\prime \prime}$, and $X_{n}^{\prime \prime \prime}$ where

$$
\begin{aligned}
X_{n}^{+} & =f\left(\left\lceil\theta_{n}\right\rceil\right)+\varepsilon_{n}^{+} \\
X_{n}^{-} & =f\left(\left\lfloor\theta_{n}\right\rfloor\right)+\varepsilon_{n}^{-} \\
X_{n}^{\prime} & =f\left(\left[\theta_{n}\right]-1\right)+\varepsilon_{n}^{\prime} \\
X_{n}^{\prime \prime} & =f\left(\left[\theta_{n}\right]\right)+\varepsilon_{n}^{\prime \prime} \\
X_{n}^{\prime \prime \prime} & =f\left(\left[\theta_{n}\right]+1\right)+\varepsilon_{n}^{\prime \prime \prime}
\end{aligned}
$$

and $\left(\varepsilon_{n}^{+}, \varepsilon_{n}^{-}, \varepsilon_{n}^{\prime}, \varepsilon_{n}^{\prime \prime}, \varepsilon_{n}^{\prime \prime \prime} ; n=1,2, \ldots\right)$ are independent and identically distributed random variables with mean zero. Let $\mathscr{F}_{1} \subset \mathscr{F}_{2} \subset \ldots$ be an increasing sequence of $\sigma$-fields such that $\varepsilon_{n}^{+}, \varepsilon_{n}^{-}, \varepsilon_{n}^{\prime}, \varepsilon_{n}^{\prime \prime}$, and $\varepsilon_{n}^{\prime \prime \prime}$ are $\mathscr{F}_{n}$-measurable and independent of $\mathscr{F}_{n-1}$ for all $n \geq 2$. Then $\theta_{n+1}$ is computed from the recursion

$$
\theta_{n+1}=\theta_{n}-\frac{c}{n} \frac{X_{n}^{+}-X_{n}^{-}}{H_{n}}
$$

where $H_{n}$ is a truncated average of $\left(X_{i}^{\prime}-2 X_{i}^{\prime \prime}+X_{i}^{\prime \prime \prime} ; i=1, \ldots, n\right)$. That is,

$$
H_{n}= \begin{cases}a, & \text { if } G_{n}<a \\ G_{n}, & \text { otherwise } \\ b, & \text { if } G_{n}>b\end{cases}
$$

where

$$
G_{n}=\frac{1}{n} \sum_{i=1}^{n}\left(X_{i}^{\prime}-2 X_{i}^{\prime \prime}+X_{i}^{\prime \prime \prime}\right) .
$$

At iteration $n$, the estimate of the optimal solution is the nearest integer to $\theta_{n}$.

Below is the detailed description of the proposed algorithm.

\section{Algorithm}

1. Set $n=1$ and choose $\theta_{n}$ randomly from $Z$.

2. Set

$$
\theta_{n+1}=\theta_{n}-\frac{c}{n} \frac{X_{n}^{+}-X_{n}^{-}}{H_{n}}
$$

3. Set $n=n+1$ and go to Step 2 .

The following assumptions will be needed:

A1. $f$ has only one local minimum at $\theta_{*}$. We say $\theta \in Z$ is a local minimum if $f(\theta) \leq f(\theta+1)$ and $f(\theta) \leq f(\theta-1)$.

A2. $\quad$ There exist known constants $a$ and $b$ such that

$$
0<a<f\left(\theta_{*}+1\right)-2 f\left(\theta_{*}\right)+f\left(\theta_{*}-1\right)<b<\infty .
$$

A3. $\quad \max \left(\operatorname{Var}\left(\varepsilon_{1}^{+}\right), \operatorname{Var}\left(\varepsilon_{1}^{-}\right), \operatorname{Var}\left(\varepsilon_{1}^{\prime}\right), \operatorname{Var}\left(\varepsilon_{1}^{\prime \prime}\right), \operatorname{Var}\left(\varepsilon_{1}^{\prime \prime \prime}\right)\right) \leq \sigma^{2}<\infty$.

A4. There exists a constant $\beta>0$ such that $|f(\theta+1)-f(\theta)| \geq \beta$ for all $\theta \in Z$.

A5. There exists a constant $C$ such that

$$
|f(\theta+1)-f(\theta)| \leq C\left(1+\left|\theta-\theta_{*}\right|\right)
$$

for all $\theta \in Z$. 


\section{Lim}

A6. $\quad c$ is large enough so that $c \gamma>\left(f\left(\theta_{*}+1\right)-2 f\left(\theta_{*}\right)+f\left(\theta_{*}-1\right)\right)$, where

$$
\gamma=\inf _{\theta \in R} \frac{|f(\lceil\theta\rceil)-f(\lfloor\theta\rfloor)|}{\left|\theta-\theta_{*}\right|}>0
$$

A7. There exists $K<\infty$ such that

$$
\max \left(\left|\varepsilon_{1}^{+}\right|,\left|\varepsilon_{1}^{-}\right|,\left|\varepsilon_{1}^{\prime}\right|,\left|\varepsilon_{1}^{\prime \prime}\right|,\left|\varepsilon_{1}^{\prime \prime \prime}\right|\right)<K
$$

and

$$
\left|\theta_{1}\right| \leq K
$$

with probability one.

We now state our main results as follows:

Theorem 1 Under Al-A5, $\theta_{n} \rightarrow \theta_{*}$ as $n \rightarrow \infty$ with probability one.

Theorem 2 Under A1-A7,

$$
\left|\theta_{n}-\theta_{*}\right|=O_{p}\left(\frac{1}{n}\right) \quad \text { as } n \rightarrow \infty
$$

\section{PROOFS}

Proof of Theorem 1 For simplicity of writing, we assume $\theta_{*}=0$. Let $A_{n}=\left(X_{n}^{+}-X_{n}^{-}\right) / H_{n}$. First, we prove

$$
\begin{aligned}
& \inf _{\varepsilon \leq\left|\theta_{n}-\theta_{*}\right| \leq 1 / \varepsilon}\left(\theta_{n}-\theta_{*}\right) E A_{n} \geq \varepsilon \beta b^{-1}>0 \text { for every } \varepsilon>0 \\
& E\left(A_{n}^{2} \mid \mathscr{F}_{n}\right) \leq C_{1}\left(1+\left(\theta_{n}-\theta_{*}\right)^{2}\right) \text { for some constant } C_{1} .
\end{aligned}
$$

For any $\theta_{n}$ with $\varepsilon \leq\left|\theta_{n}-\theta_{*}\right| \leq 1 / \varepsilon$,

$$
\begin{aligned}
& \left(\theta_{n}-\theta_{*}\right) E\left(A_{n} \mid \mathscr{F}_{n}\right) \\
& \quad=\theta_{n} E\left[\frac{X_{n}^{+}-X_{n}^{-}}{H_{n}}\right] \\
& =\theta_{n}\left(f\left(\left\lceil\theta_{n}\right\rceil\right)-f\left(\left\lfloor\theta_{n}\right\rfloor\right)\right) E\left[1 / A_{n}\right] .
\end{aligned}
$$

Note that A1 implies $f(\lceil\theta\rceil)-f(\lfloor\theta\rfloor) \geq 0$ if $\theta>0$ and $f(\lceil\theta\rceil)-f(\lfloor\theta\rfloor) \geq 0$ if $\theta \leq 0$. So, $\theta(f(\lceil\theta\rceil)-f(\lfloor\theta\rfloor))=$ $|\theta \|(f(\lceil\theta\rceil)-f(\lfloor\theta\rfloor))|$ for any $\theta \in R$. So,

$$
\begin{aligned}
\theta_{n}\left(f\left(\left\lceil\theta_{n}\right\rceil\right)-f\left(\left\lfloor\theta_{n}\right\rfloor\right)\right) E\left[1 / H_{n}\right] & =\left|\theta_{n}\right|\left|f\left(\left\lceil\theta_{n}\right\rceil\right)-f\left(\left\lfloor\theta_{n}\right\rfloor\right)\right| E\left[1 / H_{n}\right] \\
& \geq \varepsilon \beta b^{-1} \quad \text { by A4. }
\end{aligned}
$$

Hence (7) is proven. Note that

$$
\begin{aligned}
E & \left(A_{n}^{2} \mid \mathscr{F}_{n}\right) \\
& =E\left[\frac{\left(X_{n}^{+}-X_{n}^{-}\right)^{2}}{H_{n}^{2}} \mid \mathscr{F}_{n}\right] \\
& \leq\left(\left(f\left(\left\lceil\theta_{n}\right\rceil\right)-f\left(\left\lfloor\theta_{n}\right\rfloor\right)\right)^{2}+2 \sigma^{2}\right) E\left[\frac{1}{H_{n}^{2}} \mid \mathscr{F}_{n}\right] \\
& \leq b^{-2}\left(\left(f\left(\left\lceil\theta_{n}\right\rceil\right)-f\left(\left\lfloor\theta_{n}\right\rfloor\right)\right)^{2}+2 \sigma^{2}\right) \quad \text { by A2 } \\
& \leq b^{-2}\left(C^{2}\left(1+\left\lfloor\theta_{n}\right\rfloor\right)^{2}+2 \sigma^{2}\right) \text { by A5 }
\end{aligned}
$$




$$
\begin{aligned}
& \operatorname{Lim} \\
\leq & b^{-2}\left(C^{2}\left(2+\left|\theta_{n}\right|\right)^{2}+2 \sigma^{2}\right) \\
\leq & C_{1}\left(1+\left|\theta_{n}\right|^{2}\right),
\end{aligned}
$$

for some constant $C_{1}$. Hence, (8) is proven.

To prove $\theta_{n} \rightarrow 0$, note

$$
\begin{aligned}
E & \left(\theta_{n+1}^{2} \mid \mathscr{F}_{n}\right) \\
& =E\left(\left(\theta_{n}-\frac{c}{n} A_{n}\right)^{2} \mid \mathscr{F}_{n}\right) \\
& =\theta_{n}^{2}+\frac{c^{2}}{n^{2}} E\left(A_{n}^{2} \mid \mathscr{F}_{n}\right)-\frac{2 c}{n} \theta_{n} E\left(A_{n} \mid \mathscr{F}_{n}\right) \\
& \leq \theta_{n}^{2}+\frac{c^{2}}{n^{2}} C_{1}\left(1+\theta_{n}^{2}\right)-\frac{2 c}{n} \theta_{n} E\left(A_{n} \mid \mathscr{F}_{n}\right) \quad \text { by }(8) \\
& \leq\left(1+\frac{c^{2} C_{1}}{n^{2}}\right) \theta_{n}^{2}+\frac{c^{2} C_{1}}{n^{2}}-\frac{2 c}{n} \theta_{n} E\left(H\left(\theta_{n}\right) \mid \mathscr{F}_{n}\right) .
\end{aligned}
$$

By the theorem for almost supermartingales in Robbins and Siegmund (1971), $\theta_{n}^{2} \rightarrow \theta_{\infty}$ for some random variable $\theta_{\infty}$ as $n \rightarrow \infty$ and

$$
\sum_{n=1}^{\infty} \frac{1}{n} \theta_{n} E\left(A_{n} \mid \mathscr{F}_{n}\right)<\infty
$$

with probability one. We now need to show $\theta_{\infty}=0$ with probability one. Suppose, on the contrary, that $\omega$ is such that $\theta_{\infty}(\omega) \neq 0$. Then there exist $\delta>0$ and $N$ such that for all $n>N, \delta \leq \theta_{n}(\omega) \leq 1 / \delta$. Since $\theta_{n} E\left(A_{n} \mid \mathscr{F}_{n}\right) \geq \delta \beta b^{-1}>0$ for all $\delta \leq \theta_{n}(\omega) \leq 1 / \delta$ from (7), we have

$$
\sum_{n=1}^{\infty} \frac{1}{n} \theta_{n} E\left(A_{n} \mid \mathscr{F}_{n}\right)=\infty
$$

which contradicts (9). Hence $\theta_{\infty}=0$ with probability one.

Proof of Theorem 2 It suffices to prove

$$
\sup _{n=1,2, \ldots} E\left(n\left|\theta_{n}-\theta_{*}\right|\right)<\infty
$$

since for any constant $C$,

$$
P\left(n\left|\theta_{n}-\theta_{*}\right|>C\right) \leq \frac{E\left(n\left|\theta_{n}-\theta_{*}\right|\right)}{C} .
$$

For simplicity of writing, assume $\theta_{*}=0$. First assume $\theta_{1}=\theta_{*}$. (We will consider the general case later.) Let $Y_{n}=$ $n\left(\theta_{n}-\theta_{*}\right), n=1, \ldots$. We will prove that there exists $N$ such that for all $n \geq N$,

$$
E\left(Y_{n+1}-Y_{n} \mid \mathscr{F}_{n}\right) \leq-\varepsilon^{\prime} \text { on the event } Y_{n}>\theta_{*}
$$

and

$$
E\left(Y_{n+1}-Y_{n} \mid \mathscr{F}_{n}\right) \geq \varepsilon^{\prime \prime} \quad \text { on the event } Y_{n}<\theta_{*}
$$

for some constants $\varepsilon^{\prime}, \varepsilon^{\prime \prime}>0$. From

$$
\theta_{n+1}=\theta_{n}-\frac{c}{n} \frac{X_{n}^{+}-X_{n}^{-}}{H_{n}},
$$


we get

$$
(n+1) \theta_{n+1}=(n+1) \theta_{n}-c\left(1+\frac{1}{n}\right) \frac{X_{n}^{+}-X_{n}^{-}}{H_{n}}
$$

or equivalently,

$$
Y_{n+1}=Y_{n}+\theta_{n}-c\left(1+\frac{1}{n}\right) \frac{X_{n}^{+}-X_{n}^{-}}{H_{n}}
$$

When $Y_{n}>\theta_{*}$,

$$
\begin{aligned}
E & \left(Y_{n+1}-Y_{n} \mid \mathscr{F}_{n}\right) \\
& =\theta_{n}-c\left(1+\frac{1}{n}\right) E\left[\frac{X_{n}^{+}-X_{n}^{-}}{H_{n}}\right] \\
& =\theta_{n}-c\left(1+\frac{1}{n}\right)\left(f\left(\left\lceil\theta_{n}\right\rceil\right)-f\left(\left\lfloor\theta_{n}\right\rfloor\right)\right) E\left[\frac{1}{A_{n}}\right] .
\end{aligned}
$$

Under A1-A4, $\theta_{n} \rightarrow \theta_{*}$ as $n \rightarrow \infty$ with probability one by Theorem 1 , and hence $1 / A_{n} \rightarrow 1 /(f(1)-2 f(0)+f(-1))$ with probability one as $n \rightarrow \infty$. Since $1 / b \leq 1 /\left|H_{n}\right| \leq 1 / a$ for all $n$, by the bounded convergence theorem, $E\left[1 / H_{n}\right] \rightarrow$ $(f(1)-2 f(0)+f(-1))^{-1}$ as $n \rightarrow \infty$. Take $\varepsilon$ small enough so that

$$
\gamma c(f(1)-2 f(0)+f(-1))^{-1}-1>c \gamma \varepsilon
$$

Then there exists $N$ such that for all $n \geq N$,

$$
\mid E\left[1 / H_{n}\right]-\left((f(1)-2 f(0)+f(-1))^{-1} \mid \leq \varepsilon\right.
$$

For $n \geq N$,

$$
\begin{aligned}
\theta_{n} & -c\left(1+\frac{1}{n}\right)\left(f\left(\left\lceil\theta_{n}\right\rceil\right)-f\left(\left\lfloor\theta_{n}\right\rfloor\right)\right) E\left[\frac{1}{H_{n}}\right] \\
& \left.\leq \theta_{n}-c\left(1+\frac{1}{n}\right)(f(1)-2 f(0)+f(-1))^{-1}-\varepsilon\right)\left(f\left(\left\lceil\theta_{n}\right\rceil\right)-f\left(\left\lfloor\theta_{n}\right\rfloor\right)\right) \\
& \left.\leq\left\lceil\theta_{n}\right\rceil-c\left(1+\frac{1}{n}\right)(f(1)-2 f(0)+f(-1))^{-1}-\varepsilon\right)\left(f\left(\left\lceil\theta_{n}\right\rceil\right)-f\left(\left\lfloor\theta_{n}\right\rfloor\right)\right) \text { because } \theta_{n}>0 \\
& \left.\leq\left\lceil\theta_{n}\right\rceil-c(f(1)-2 f(0)+f(-1))^{-1}-\varepsilon\right)\left(f\left(\left\lceil\theta_{n}\right\rceil\right)-f\left(\left\lfloor\theta_{n}\right\rfloor\right)\right) \\
& \leq\left\lceil\theta_{n}\right\rceil-c\left((f(1)-2 f(0)+f(-1))^{-1}-\varepsilon\right) \gamma\left\lceil\theta_{n}\right\rceil \text { by A6 } \\
& =\left\lceil\theta_{n}\right\rceil\left(1-c(f(1)-2 f(0)+f(-1))^{-1} \gamma+c \varepsilon \gamma\right) \\
& \leq-\varepsilon^{\prime}\left\lceil\theta_{n}\right\rceil \text { for some } \varepsilon^{\prime}>0 \quad \text { by }(12) \\
& \leq-\varepsilon^{\prime} \text { because }\left\lceil\theta_{n}\right\rceil \geq 1 \text { for all } \theta_{n}>0 .
\end{aligned}
$$

Hence (10) holds. (11) follows in a similar way.

Let $\mu_{i}=E\left((i+1) \theta_{i+1}-i \theta_{i} \mid \mathscr{F}_{i}\right)$ for $i=1,2, \ldots$. Note that $\mathrm{A} 7$ implies

$$
\left|\mu_{i}\right| \leq b(K, N)
$$

for all $i=1, \ldots, N$ with probability one where $b(K, N)$ is a constant depending on $K$ and $N$ because

$$
\begin{aligned}
\left|\mu_{i}\right| & =\left|E\left((i+1) \theta_{i+1}-i \theta_{i} \mid \mathscr{F}_{i}\right)\right| \\
& =E\left(\left|(i+1) \theta_{i+1}-i \theta_{i}\right| \mid \mathscr{F}_{i}\right)
\end{aligned}
$$




$$
\begin{aligned}
& \operatorname{Lim} \\
& =E\left(\left|\theta_{i}-c\left(1+\frac{1}{i}\right) \frac{X_{i}^{+}-X_{i}^{-}}{H_{i}}\right| \mid \mathscr{F}_{i}\right) \\
& \leq\left|\theta_{i}\right|+c\left(1+\frac{1}{i}\right) E\left(\frac{\left|X_{i}^{+}-X_{i}^{-}\right|}{\left|H_{i}\right|} \mid \mathscr{F}_{i}\right) \\
& \leq\left|\theta_{i}\right|+c\left(1+\frac{1}{i}\right) a^{-1}\left(f\left(\left\lceil\theta_{i}\right\rceil\right)-f\left(\left\lfloor\theta_{i}\right\rfloor\right)+2 K\right) \\
& \leq\left|\theta_{i}\right|+c\left(1+\frac{1}{i}\right) a^{-1}\left(C\left(1+\left\lceil\theta_{i}\right\rceil\right)+2 K\right) \\
& \leq C_{2}\left(1+\left|\theta_{i}\right|\right)
\end{aligned}
$$

for some constant $C_{2}$ and

$$
\begin{aligned}
\left|\theta_{i}\right| & =\left|\theta_{i-1}-\frac{c}{i-1} \frac{X_{i-1}^{+}-X_{i-1}^{-}}{H_{i-1}}\right| \\
& \leq\left|\theta_{i-1}\right|+\frac{C_{3}}{i-1}\left(1+\left|\theta_{i-1}\right|\right) \\
& \leq\left(1+\frac{C_{3}}{i-1}\right)\left|\theta_{i-1}\right|+\frac{C_{3}}{i-1} \\
& \leq \ldots \leq\left(1+\frac{C_{3}}{i-1}\right)\left(1+\frac{C_{3}}{i-2}\right) \ldots\left(1+\frac{C_{3}}{1}\right)\left|\theta_{1}\right|+\sum_{k=1}^{i-1} \prod_{j=1}^{k-1}\left(1+\frac{C_{3}}{i-j}\right) \frac{C_{3}}{i-k},
\end{aligned}
$$

for come constant $C_{3}$.

We will compute an upper bound for $E\left|Y_{n}\right|$ that does not depend on $n$. Let $U=\max \left\{k \leq n: Y_{k} \leq 0\right\}$ denote the last time up to $n$ such that $\left\{Y_{k}\right\}_{k=1, \ldots}$ takes a nonpositive value.

For $t>N \cdot b(K, N)$, we have

$$
\begin{aligned}
P\left(Y_{n} \geq t\right) & \\
= & \sum_{k=1}^{n-1} P\left(Y_{n} \geq t, U=k\right) \\
\leq & \sum_{k=1}^{n-1} P\left(Y_{n}-Y_{k} \geq t, Y_{k} \leq 0, Y_{i}>0 \text { for } k<i<n\right) \\
\leq & \sum_{k=1}^{N-1} P\left(Y_{n}-Y_{n-1}-\mu_{n-1}+\ldots+Y_{k+1}-Y_{k}-\mu_{k} \geq t+\varepsilon(n-N)-b(K, N)(N-k)\right) \\
& +\sum_{k=N}^{n-1} P\left(Y_{n}-Y_{n-1}-\mu_{n-1}+\ldots+Y_{k+1}-Y_{k}-\mu_{k} \geq t+\varepsilon(n-k)\right) \\
\leq & \sum_{k=1}^{N-1} \frac{E\left|Y_{n}-Y_{n-1}-\mu_{n-1}+\ldots+Y_{k+1}-Y_{k}-\mu_{k}\right|^{p}}{|t+\varepsilon(n-N)-b(K, N)(N-k)|^{p}} \\
& +\sum_{k=N}^{n-1} \frac{E\left|Y_{n}-Y_{n-1}-\mu_{n-1}+\ldots+Y_{k+1}-Y_{k}-\mu_{k}\right|^{p}}{|t+\varepsilon(n-k)|^{p}}
\end{aligned}
$$

for any $p>6$.

Note that $\left(Y_{n+1}-Y_{n}-\mu_{n}: n=1, \ldots\right)$ is a sequence of a martingale difference with $\sup _{n}\left|Y_{n+1}-Y_{n}-\mu_{n}\right|^{p}<\infty$ because

$$
\begin{aligned}
& E\left|Y_{n+1}-Y_{n}-\mu_{n}\right|^{p} \\
& \quad=E\left|c\left(1+\frac{1}{n}\right) \frac{X_{n}^{+}-X_{n}^{-}}{H_{n}}-c\left(1+\frac{1}{n}\right) E\left(\frac{X_{n}^{+}-X_{n}^{-}}{H_{n}}\right)\right|^{p}
\end{aligned}
$$




$$
\begin{aligned}
& \operatorname{Lim} \\
& =c^{p}\left(1+\frac{1}{n}\right)^{p} E\left|\frac{X_{n}^{+}-X_{n}^{-}}{H_{n}}-E\left(\frac{X_{n}^{+}-X_{n}^{-}}{H_{n}}\right)\right|^{p} \\
& \leq c^{p} 2^{p} E\left|\frac{X_{n}^{+}-X_{n}^{-}-\left(f\left(\left\lceil\theta_{n}\right\rceil\right)-f\left(\left\lfloor\theta_{n}\right\rfloor\right)\right) E\left(1 / H_{n}\right) H_{n}}{H_{n}}\right|^{p} \\
& \leq c^{p} 2^{p} b^{-p} E C_{4}\left(1+\left|\theta_{n}\right|\right)^{p} \\
& \leq c^{p} 2^{p} b^{-p}\left(C_{4}+E\left|\theta_{n}\right|^{p}\right)
\end{aligned}
$$

for some constant $C_{4}$ and

$$
\begin{aligned}
E\left|\theta_{n+1}\right|^{p} & =E\left|\theta_{n}-\frac{c}{n} \frac{X_{n}^{+}-X_{n}^{-}}{H_{n}}\right|^{p} \\
& \leq E\left|\theta_{n}\right|^{p}+\frac{c^{p}}{n^{p}} b^{-p} C_{5}\left(1+E\left|\theta_{n}\right|^{p}\right) \\
& =\left(1+\frac{C_{5}}{n^{p}}\right) E\left|\theta_{n}\right|^{p}+\frac{C_{5}}{n^{p}}
\end{aligned}
$$

for some constants $C_{5}$ and $C_{6}$. Hence, by Lemma 1 of Venter (1966), $E\left|\theta_{n}\right|^{p}$ is bounded.

Note

$$
\sum_{k=N}^{n-1} \frac{E\left|Y_{n}-Y_{n-1}-\mu_{n-1}+\ldots+Y_{k+1}-Y_{k}-\mu_{k}\right|^{p}}{|t+\varepsilon(n-k)|^{p}} \leq \sum_{k=N}^{n-1} \frac{C_{p}(n-k)^{p / 2}}{(t+\varepsilon(n-k))^{p}} \leq \sum_{k=1}^{\infty} \frac{C_{p} k^{p / 2}}{(t+\varepsilon k)^{p}}
$$

and that

$$
\sum_{k=1}^{N-1} \frac{E\left|Y_{n}-Y_{n-1}-\mu_{n-1}+\ldots+Y_{k+1}-Y_{k}-\mu_{k}\right|^{p}}{|t+\varepsilon(n-N)-b(K, N) N|^{p}} \leq \sum_{k=1}^{N-1} \frac{C_{p}(n-k)^{p / 2}}{|t+\varepsilon(n-N)-b(K, N) N|^{p}}
$$

for some constant $C_{p}$ by Lemma 2.1 of $\mathrm{Li}$ (2003). So,

$$
\begin{aligned}
E \max \left(Y_{n}, 0\right)= & \int_{0}^{\infty} P\left(Y_{n}>t\right) d t \\
= & N \cdot b(K, N)+\int_{N \cdot b(K, N)}^{\infty} \sum_{k=1}^{\infty} \frac{C_{p} k^{p / 2}}{(t+\varepsilon k)^{p}} \\
& \quad+\int_{N \cdot b(K, N)}^{\infty} \sum_{k=1}^{N-1} \frac{C_{p}(n-k)^{p / 2}}{|t+\varepsilon(n-N)-b(K, N) N|^{p}} \\
= & N \cdot b(K, N)+\sum_{k=1}^{\infty} \int_{b(K, N)}^{\infty} \frac{C_{p} k^{p / 2}}{(t+\varepsilon k)^{p}} \\
& \quad+\sum_{k=1}^{N-1} \int_{N \cdot b(K, N)}^{\infty} \frac{C_{p}(n-k)^{p / 2}}{|t+\varepsilon(n-N)-b(K, N) N|^{p}} \\
= & N \cdot b(K, N)+\sum_{k=1}^{\infty} \frac{1}{p-1} \frac{C_{p} k^{p / 2}}{|N \cdot b(K, N)+\varepsilon k|^{p-1}} \\
& \quad+\sum_{k=1}^{N} \frac{1}{p-1} \frac{C_{p}(n-k)^{p / 2}}{|N \cdot b(K, N)+\varepsilon(n-N)-b(K, N) N|^{p}} \\
\leq & K^{\prime}
\end{aligned}
$$

for some constant $K^{\prime}<\infty$ which does not depend on $n . E \max \left(-Y_{n}, 0\right)<\infty$ follows in a similar way. Hence the desired result is proven. For the general case, let $Y_{n}=n\left(\theta_{n}-\theta_{*}\right)-\left(\theta_{1}-\theta_{*}\right)$. Then it follows $E\left(n\left(\theta_{n}-\theta_{*}\right)-\left(\theta_{1}-\theta_{*}\right)\right)^{+}<\infty$ and 
$E\left(n\left(\theta_{n}-\theta_{*}\right)-\left(\theta_{1}-\theta_{*}\right)\right)^{-}<\infty$. So

$$
E\left|n\left(\theta_{n}-\theta_{*}\right)\right| \leq E\left|n\left(\theta_{n}-\theta_{*}\right)-\theta_{1}\right|+E\left|\theta_{1}\right|+\theta_{*}<\infty
$$

by A7. Hence, the desired result is proven.

\section{A NUMERICAL EXAMPLE}

Consider the single-period newsvendor problem where $\theta$ units are ordered and stacked at the beginning of the period. The goal is to find an ordering level $\theta$ that minimizes the cost function $f(\theta)=E[c \theta+h \max (0, \theta-D)+p \max (0, D-\theta)]$, where $c$ is the unit cost for producing each unit, $h$ is the holding cost per unit remaining at the end of the period, $p$ is the shortage cost per unit of unsatisfied demand, and the expectation is taken with respect to the random demand $D$. The optimal solution for this problem is given by

$$
\theta_{*}=F^{-1}\left(\frac{p-c}{p+h}\right),
$$

where $F$ is the cumulative distribution function of $D$.

We compare the proposed algorithm:

$$
\theta_{n+1}=\theta_{n}-\frac{1}{n} \frac{X_{n}^{+}-X_{n}^{-}}{A_{n}}
$$

to the following algorithm:

$$
\theta_{n+1}=\theta_{n}-\frac{1}{n}\left(X_{n}^{+}-X_{n}^{-}\right)
$$

which was proposed in Lim and Glynn (2006) and which does not make use of the Hessian information.

Table 1 compares the performance of (14) and (15) with $c=3, h=5$, and $p=9$. Demand follows a Poisson distribution with parameter 100 , resulting in $\theta_{*}=98$. In (14) and (15), $1 / n$ is adjusted according to the total number of the iterations as follows:

\section{Total number of iterations}

so that $a_{n} \in\{1 / 1,1 / 2,1 / 3, \ldots, 1 / 10\}$ and $a_{n}$ does not get too small at the end of iterations.

At each $\theta_{n}, X_{n}^{+}$is the average of 500 replications of $c\left\lceil\theta_{n}\right\rceil+h\left(\left\lceil\theta_{n}\right\rceil-D\right)^{+}+p\left(D-\left\lceil\theta_{n}\right\rceil\right)^{+} . X_{n}^{-}, X_{n}^{\prime}, X_{n}^{\prime \prime}$, and $X_{n}^{\prime \prime \prime}$ are computed in a similar way. Table 1 shows the sample mean and the sample standard deviation of $\theta_{n}$ based on 200 independent replications with $\theta_{1}=5.231 . n_{1}$ and $n_{2}$ are the total numbers of iterations for (14) and (15), respectively. The ratio of $n_{1}$ to

\begin{tabular}{|c|c|c|c|c|c|c|c|c|c|}
\hline & \multicolumn{3}{|c|}{$n_{1}=15, n_{2}=6$} & \multicolumn{3}{|c|}{$n_{1}=25, n_{2}=10$} & \multicolumn{3}{|c|}{$n_{1}=35, n_{2}=14$} \\
\hline$\theta_{*}=98$ & $\mid \theta_{n}-\theta_{*}$ & Variance & MSE & $\overline{\mid \theta_{n}-\theta_{*}}$ & Variance & MSE & $\overline{\left|\theta_{n}-\theta_{*}\right|}$ & Variance & MSE \\
\hline Algorithm (14) & 51.14 & 1003.00 & 3618.29 & 24.00 & 967.21 & 1543.21 & 18.14 & 907.21 & 1236.27 \\
\hline Algorithm (15) & 69.31 & 73.49 & 4877.32 & 50.94 & 106.92 & 2701.80 & 33.69 & 153.76 & 1288.78 \\
\hline
\end{tabular}
$n_{2}$ is set to be 2 to 5 reflecting the fact that we need to generate $X(\theta)$ at 5 and 2 different values of $\theta$ at each iteration of (14) and (15), respectively. Hence the same amount of computational budget is allocated to (14) and (15).

Table 1: Performance of Algorithms (14) and (15)

Table 1 shows that the proposed algorithm, Algorithm (14), approaches the optimal solution faster than Algorithm (15), but shows more variability. However, the overall efficiency summarized by the mean square error indicates that the proposed algorithm outperforms Algorithm (15) since the variance increase is more than offset by the bias reduction. 


\section{ACKNOWLEDGMENTS}

The author would like to thank all anonymous referees for their valuable comments and suggestions.

\section{REFERENCES}

Andradottir, S. 1995. A method for discrete stochastic optimization. Management Science 41:1946-1961.

Chung, K. L. 1954. On a stochastic approximation method. Annals of Mathematical Statistics 25:463-483.

Dupăc, V., and U. Herkenrath. 1982. Stochastic approximation on a discrete set and the multi-armed bandit problem. Communications in Statistics-Sequential Analysis 1:1-25.

Gelfand, S., and S. Mitter. 1989. Simulated annealing with noisy or imprecise energy measurements. Journal of Optimization Theory and Applications 2:49-62.

Hong, L. J., and B. L. Nelson. 2006. Discrete optimization via simulation using compass. Operations Research 54:115-129.

Kleywegt, A., A. Shapiro, and T. Homem-de-Mello. 2001. The sample average approximation for stochastics discrete optimization. SIAM Journal on Optimization 12:479-502.

Li, Y. 2003. A martingale inequality and large deviations. Statistics and Probability Letters 62:317-321.

Lim, E., and P. W. Glynn. 2006. Discrete optimization via simulation in the presence of regularity. INFORMS National Meeting, Pittsburgh.

Lin, X., and L. H. Lee. 2006. A new approach to discrete stochastic optimization problems. European Journal of Operational Research 172:761-782.

Robbins, H., and S. Monro. 1951. A stochastic approximation method. Annals of Mathematical Statistics 22:400-407.

Robbins, H., and D. Siegmund. 1971. A convergence theorem for nonnegative almost supermartingales and some applications. In Optimizing Motheds in Statistics, 233-257. New York: Academic Press.

Shi, L., and S. Olafsson. 2000. Nested partitions method for stochastic optimization. Methodology and Computing in Applied Probability 2:271-291.

Venter, J. H. 1966. On Dvoretzky stochastic approximation theorems. Annals of Mathematical Statistics 37:1534-1544.

\section{AUTHOR BIOGRAPHY}

EUNJI LIM is an Assistant Professor in the Industrial Engineering Department at the University of Miami. She received her Ph.D. in Management Science and Engineering from Stanford University. Her research interest includes stochastic optimization, statistical inference under shape restrictions, and simulation. 